\title{
Guideline for medical certification of death in the COVID-19 era
}

COVID-19 was declared a pandemic by the World Health Organization (WHO) in March 2020 $0^{[1]}$ following an outbreak of a pneumonia of unknown aetiology associated with a novel coronavirus (SARS-CoV-2) in Wuhan City, Hubei Province of China. ${ }^{[2]}$ There are currently over 8.6 million cases of COVID-19 worldwide with over 450000 deaths $^{[3]}$ - and still counting. The pandemic is gradually accelerating in Africa, with more than 200000 cases and currently over 5600 deaths, only five countries (Algeria, Egypt, Nigeria, South Africa (SA) and Sudan) accounting for more than 70\% of the deaths. ${ }^{[4]}$ In SA, COVID-19 had resulted in over 2900 deaths (over 175000 cases $)^{[5]}$ by the beginning of July.

With increasing mortality from COVID-19, it is essential to undertake proper counting of the deaths in order to plan and provide adequate public health strategies for dealing with the present crisis, preventing future crises and tracking changes over time. Information from death certificates can be used for epidemic surveillance, outbreaks and emergencies, such as the COVID-19 pandemic. ${ }^{[6]}$ In SA, rapid reporting of deaths is required by law according to the Births and Deaths Registration Act 51 of 1992. ${ }^{[7]}$ Cause of death information is used to indicate community health concerns and priorities. Furthermore, it alerts local and national agencies including the healthcare community at large to epidemics and severe medical complications of infectious and environmental exposures. Accurate and complete reporting of cause of death ensures that surveillance is effective. If it is not effective, it can be disastrous, as was the case previously with reporting HIV as cause of death in SA. ${ }^{[8]}$

The national cause of death statistics are compiled from information provided by doctors on the medical certificate of cause of death section of the death notification form (DHA-1663). For each deceased person, a doctor is required to provide details of the sequence of diseases terminating in death or causal sequence, such that the underlying cause of death can be identified. ${ }^{[9]}$

The WHO recently provided international guidelines on the medical certification of cause of death due to COVID-19. ${ }^{[10,11]}$ These indicate that COVID-19 should be recorded on the medical certificate of cause of death for all decedents where COVID-19 caused, or is assumed to have caused, or contributed to death.

Until testing for SARS-CoV-2 is more accessible, mortality due to COVID-19 is the only population-based measure of the epidemic. For this reason, it is imperative that clinicians in SA are aware of their obligation under law ${ }^{[7]}$ to report accurate causes of death on the SA death notification form. This means that clinicians are bound by law to report COVID-19 as a cause of death in cases where this has been confirmed, as well as in cases where, in their opinion, it is the most probable cause of death, even if a test result is not available to confirm this. To facilitate accurate certification of cause of death in SA, the SA WHO-FIC Collaborating Centre developed a short local guideline ${ }^{[12]}$ based on the WHO international guidelines. This has been reviewed by the Ministerial Advisory Committee on COVID-19 and recommended to the National Department of Health for adoption.

\section{Definition of a death due to COVID- 19 (based on WHO ICD-10)}

A death due to COVID-19 is defined for surveillance purposes as a death resulting from an illness that is compatible with COVID-19 (based on laboratory confirmation or on clinical features only). There should be no period of complete recovery from COVID-19 between illness and death.

\section{Certification of a death due to COVID-19}

The underlying cause of death is defined as 'The disease or injury which initiated the train of morbid events leading directly to death, or the circumstances of the accident or violence which produced the fatal injury.[. ${ }^{\text {[13] }}$ The DHA-1663 form has a cause of death section consisting of two parts: part 1 with lines a) to d) includes the causal sequence that directly led to death, with the immediate cause of death entered on line a), the underlying cause of death that initiated the causal sequence on the lowest completed line, and the antecedent or intermediate cause(s) of death (if present) on the lines between the immediate and underlying cause of death. All conditions reported in part 1 should follow a logical pathophysiological causal sequence. Part 2 contains any other significant conditions contributing to death but that are not part of the causal sequence.

The underlying cause of death of a person who has been diagnosed as suffering from COVID-19 (either on the basis of laboratory confirmation or on clinical grounds only) should be stated as COVID19 in part 1 of the medical certificate of cause of death, unless a clear alternative or unrelated supervening medical cause of death (such as trauma or poisoning) is apparent. It is important to use the standard WHO terminology, COVID-19, on the medical certificate of cause of death where COVID-19 has caused or contributed to death. Certifiers should include as much detail as possible based on their knowledge of the case, from medical records or laboratory testing (e.g. COVID-19 confirmed). See Box 1 for COVID-19 case definition.

For example, where COVID-19 causes pneumonia and acute respiratory distress that results in death, all three conditions should be captured in part 1 . If the certifier has knowledge of a laboratory confirmation, 'Confirmed COVID-19' (underlying cause of death) should be entered on line c), 'Pneumonia' (antecedent or intermediate cause) on line b), and 'Acute respiratory distress' (immediate cause of death) on line a) (Fig. 1).

However, if there was no laboratory confirmation, 'Suspected COVID-19' should be entered on line c) as the underlying cause of death (Fig. 2).

The death of a person in whom COVID-19 has been diagnosed (clinically or by laboratory confirmation) but who suffers from advanced pre-existing disease (such as severe cardiovascular disease or malignancy) should be carefully considered and clinical discretion should be used to determine the underlying cause of death. If the preexisting condition/disease is then still considered to be the underlying cause of death, but COVID-19 may have played a significant role in the death of the patient, COVID-19 and the pre-existing condition should both be clearly stated in the causal sequence leading to death (in part 1 of the medical certificate). This contradicts current WHO recommendations and reflects the difficulties of selecting a single underlying cause of death. However, it allows clinicians to use their discretion in cases with advanced pre-existing disease where COVID19 plays more of a role as an immediate cause of death rather than an underlying cause.

Where pre-existing conditions are controlled and/or in the background, report these in part 2 of the medical certificate of cause of death. For example, where COVID-19 causes fatal acute respiratory acidosis in a decedent with pre-existing chronic obstructive pulmonary disease and hypertension. COVID-19 and acute respiratory acidosis should be captured in part 1 . If the certifier has the knowledge of a laboratory confirmation, 'Confirmed COVID- 


\section{Box 1. Case definition for COVID-19}

\section{Confirmed case}

A person with laboratory confirmation of SARS-CoV-2 infection (PCR), irrespective of clinical signs and symptoms.

\section{Probable case or COVID PUI}

- Persons with acute respiratory illness with sudden onset of at least one of the following: cough, sore throat, shortness of breath, anosmia or dysguesia with or without fever (history or measured) and a clinical course compatible with COVID-19, with or without the presence of comorbid conditions

- Pneumonia with bilateral patchy ground glass opacities on chest radiograph

$$
\text { AND/OR }
$$

- ARDS

AND

- Pending or inconclusive PCR result.

\section{Suspected case}

- Persons at high risk for COVID-19 who die outside a health facility or in an ER with a history of acute respiratory illness with sudden onset of at least one of the following: cough, sore throat, shortness of breath or fever.

AND

- Recent history of

- Close contact with COVID-19 case

OR

- Visit to health facility

OR

- Cluster outbreak (e.g. residential institution, care home, workplace, etc.).

$\mathrm{PCR}$ = polymerase chain reaction; $\mathrm{PUI}=$ person under investigation; $\mathrm{ARDS}$ = acute respiratory distress syndrome; $\mathrm{ER}$ = emergency room.

19 ' (underlying cause of death) should be entered on line b), and 'Acute respiratory acidosis' (immediate cause of death) on line a) (Fig. 3). Chronic obstructive pulmonary disease and hypertension should be captured in part 2 as they may have contributed to the death but are not part of the causal sequence in part 1.

COVID-19 should not be stated on the death certificate if there is a clear alternative cause of death, in which COVID-19 played no causal or contributory role (such as trauma or poisoning). COVID-19 should also not be stated as cause of death if there has been a period of complete recovery from the disease.

\section{Conclusions}

With increasing mortality from COVID-19, it is essential to undertake proper counting of the deaths in order to better understand the impact of the epidemic. Information from death certificates can be used for epidemic surveillance, outbreaks and emergencies, such as the COVID-19 pandemic. Accurate and complete reporting of cause of death ensures that surveillance is effective.

Acknowledgement. We appreciate the consideration given to these guidelines by the Ministerial Advisory Committee on COVID-19 led by Prof. Salim Abdool Karim and would like to acknowledge the advice that we received from Profs Glenda Gray, Marc Mendelsohn, Lucille Blumberg and Gert Saayman and Dr Angelique

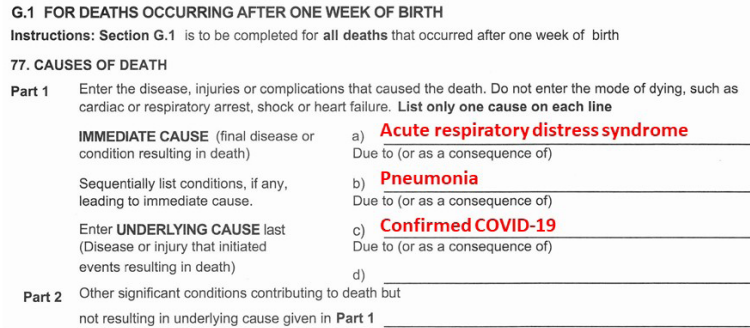

Fig. 1. Death due to COVID-19 (confirmed).

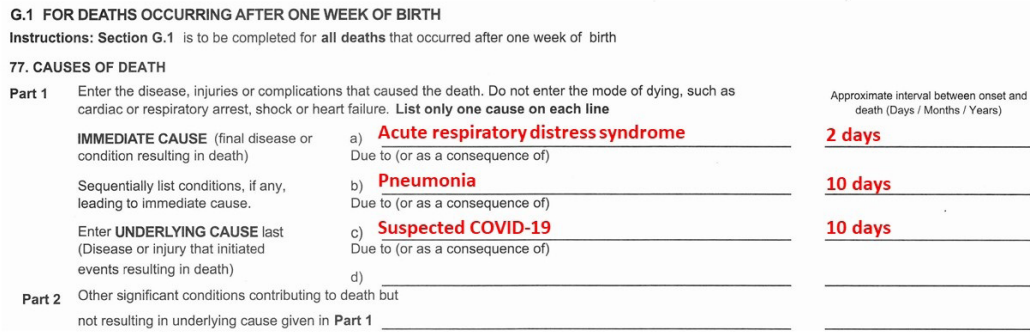

2 days

10 days

10 days

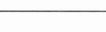

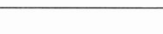

Fig. 2. Death due to COVID-19 (suspected).

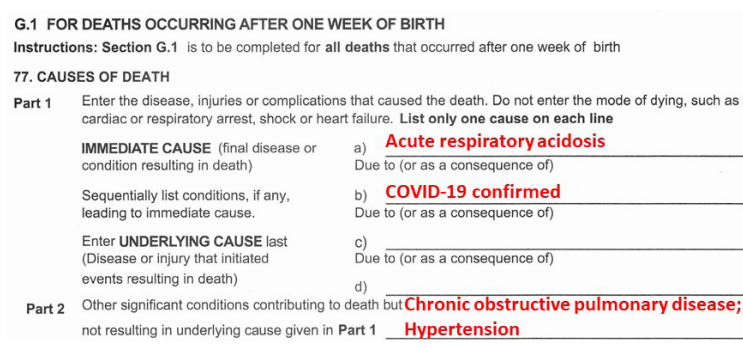
e 
Coetzee. The authors have been fully funded by the South African Medical Research Council.

\section{Pamela Groenewald, Oluwatoyin Awotiwon, Lyn Hanmer,} Debbie Bradshaw

South African WHO-FIC Collaborating Centre, South African Medical Research Council Burden of Disease Research Unit, South Africa pamela.groenewald@mrc.ac.za

1. World Health Organization. WHO Director-General's opening remarks at the media briefing on COVID-19. 11 March 2020. https://www.who.int/dg/speeches/detail/who-directorgeneral-s-openingremarks-at-the-media-briefing-oncovid-19 (accessed 19 June 2020),

. World Health Organization. Novel Coronavirus (2019-nCoV) Situation Report - 1, 21 January 2020. 2020. https://www.who.int/docs/default-source/coronaviruse/situation-reports/20200121-sitrep-1-2019ncov.pdf (accessed 19 June 2020).

3. Worldometer. COVID-19 coronavirus pandemic. https://www.worldometers.info/coronavirus/ (accessed 19 June 2020).

4. World Health Organization. Rolling updates on coronavirus disease (COVID-19) 2020. https:// www.who.int/emergencies/diseases/novel-coronavirus-2019/events-as-they-happen (accessed 19 June 2020).
5. National Institute for Infectious Diseases. National COVID-19 daily report. 3 July 2020. https://www. nicd.ac.za/wp-content/uploads/2020/06/COVID19-Daily-Report-National-Public-03July2020.pdf (accessed 4 July 2020).

6. Rao C. Medical certification of cause of death for COVID-19. Bull World Health Organ 2020;98:298-A https://doi.org/10.2471/BLT.20.257600

7. South Africa. Births and Deaths Registration Act No. 51, 1992. https://www.gov.za/sites/default/files/ gouth Africa. Births and Deaths Registration Act No. 51,
gcis_document/201409/a511992.pdf (accessed 14 July 2020).

Bradshaw D, Groenewald P. Laubscher R, et al. Initial burden of disease estimates for South Africa, 2000. Bradshaw D, Groenewald P, Laubst

9. Burger EH, Groenewald P, Rossouw A, Bradshaw D. Medical certification of death in South Africa moving forward. S Afr Med J 2015;105(1):27-30. https://doi.org/10.7196/SAMJ.8578

10. World Health Organization. International Guidelines for Certification and Classification (Coding) of COVID-19 as a Cause of Death. Based on ICD International Statistical Classification of Diseases. https:// www.who.int/classifications/icd/Guidelines_Cause_of_Death_COVID-19.pdf (accessed 19 June 2020). 1. World Health Organization. Medical certification, ICD mortality coding, and reporting mortality associated with COVID-19. 7 June 2020. https://www.who.int/publications/ $/ /$ item/WHO-2019-nCoVmortality-reporting-2020-1 (accessed 14 July 2020).

2. South African WHO-FIC Collaborating Centre. Guideline for Medical Certification of deaths due to COVID-19. May 2020. http://whoficsa.mrc.ac.za/icdresourses.html (accessed 4 July 2020).

3. World Health Organization. International Statistical Classification of Diseases and Related Healt Problems - 10th revision. 5th ed. Geneva: WHO, 2011. https://icd.who.int/browse10/Content/statichtml/ ICD10Volume2_en_2010.pdf (accessed 14 July 2020).

S Afr Med J 2020;110(8):721-723. https://doi.org/10.7196/SAMJ.2020.v110i8.15114 